\title{
La disfunción sexual femenina: ¿verdadero problema de salud 0 estrategia de marketing para posicionar un fármaco?
}

Female sexual dysfunction: real health problem or disease mongering?

Débora Ávila Poletti ${ }^{\ddagger}$, Pedro Benozzi ${ }^{\ddagger}$, Julián Feder ${ }^{\ddagger}, F$ acundo Serra ${ }^{\ddagger},{\text { María Belén Vallecillo }{ }^{\ddagger} \text { y Sergio Terrasa }}^{\S}$

Avila Poletti DB, Benozzi PG, Feder JM, Serra Aguirre FE, Vallecillo Molenberg MB, Terrasa S. La disfunción sexual femenina: ¿verdadero problema de salud o estrategia de marketing para posicionar un fármaco?. Evid Act Pract Ambul. $2015 ; 18(4): 102-103$. Oct-Dic.

\section{La promoción de enfermedades}

La ciencia y le tecnología de la salud vienen ganando terreno en el abordaje y el alivio de problemas que antes eran considerados parte de la vida, y hoy en día gozan de una enorme confianza por parte de los consumidores.

Es en ese contexto que el proceso de promoción de enfermedades (en inglés "disease mongering") cobra relevancia. Este concepto procura representar al proceso a través del cual algunas situaciones de la vida cotidiana, como por ejemplo la falta de deseo sexual en algún momento de la vida o situaciones fisiológicas como la calvicie o la pérdida de densidad ósea en edades avanzadas, son convertidas en "enfermedades" pasibles de ser abordadas por algún tratamiento**.

Para que sea eficaz la creación de la nueva enfermedad, dicha situación fisiológica o normal debe ser redefinida como un "trastorno" que merezca atención y preocupación por parte del público (potencial consumidor).

Una vez "informado" el público y procurando que los potenciales clientes desarrollen una "conciencia" colectiva de que algo anda mal, continúa usándose la publicidad con el objetivo de convertirlos en nuevos pacientes y posibles consumidores de los productos en cuestión. No es menor el rol de la "Institución médica", que se va convirtiendo en un articulador de la prescripción y el consumo de estos productos promovidos por las industrias farmacéuticas, como una suerte de "mediador" entre la oferta y la demanda. Vale destacar que cuando un paciente consulta a un profesional de la salud espera que éste le brinde un consejo que maximice las chances de beneficio respecto de las alternativas disponibles para enfrentar su sufrimiento, sin embargo, quién debería comportarse como árbitro -o por lo menos como un consejero al servicio de su cliente-, algunas veces actúa como cómplice del patrocinador del producto en venta, sin que este vínculo sea transparente como en otras transacciones comerciales en la que podemos llegar a involucrarnos ${ }^{\text {tt }}$.

\section{La disfunción sexual femenina}

La disfunción sexual femenina ha sido analogada al "Trastorno del interés/excitación sexual femenino" descripto en la quinta edición del Manual Diagnóstico y Estadístico de Trastornos Mentales (DSM V) ${ }^{1}$ de la Asociación de Psiquiatría de EE.UU. Esta entidad se refiere a una deficiencia persistente o recurrente de deseo de actividad sexual y fantasías sexuales que causan notable angustia o dificultades interpersonales ${ }^{2}$.

\section{La flibanserina}

Recientemente aprobada por la Administración de Drogas y Alimentos (FDA) de EE.UU, la Flibanserina (Addyi ${ }^{\circledR}$ ) tiene un mecanismo exacto de acción desconocido. Actuaría en forma parecida a un antidepresivo, ya que ejerce su acción a nivel central, modificando las vías de la dopamina y la serotonina.

La efectividad de una dosis de $100 \mathrm{mg}$ de flibanserina administrada a la hora de dormir fue evaluada en tres ensayos clínicos aleatorizados controlados contra placebo, que incluyeron un total de 2.400 mujeres (edad promedio 36 años) con una duración promedio de sus síntomas de DSF de cinco años. Todos los meses las mujeres completaron un cuestionario que evaluaba su deseo, su estimulación y satisfacción sexual, los orgasmos obtenidos, su lubricación en la intimidad y si habían tenido dolor. El resultado primario fue el número reportado de eventos sexuales satisfactorios en el transcurso de las cuatro semanas previas, y el de incomodidad vinculada a un bajo deseo sexual (puntaje de 0 a 4).

Las pacientes que recibieron flibanserina reportaron un promedio de 0,5 a 1 eventos sexuales satisfactorios por mes más que las que recibieron placebo; 0,35 puntos menos en promedio de incomodidad vinculada al bajo deseo sexual (rango del puntaje de 0 a 5) y una mejoría global en la satisfacción de sus eventos sexuales atribuible al tratamiento del $10 \%{ }^{3}$.

En las asignadas a placebo la mejoría global del cuestionario fue de $3,8 \%$ respecto del puntaje basal y en las asignadas a flibanserina, $4,4 \%(0,6 \% \text { de beneficio atribuible al tratamiento })^{4}$.

Vale destacar que la aprobación de la flibanserina tardó varios años ya que en un principio (entre 2010 y 2013) había sido rechazada dos veces por la FDA por su escasa eficacia comparada con placebo ${ }^{4}$ (ver párrafo anterior), y además, por sus efectos adversos.

En este sentido, un ensayo clínico que había comparado a la flibanserina contra placebo en 3.000 mujeres premenopáusicas documentó que las participantes que recibieron flibanserina experimentaron mareos, somnolencia, náuseas, fatiga, insomnio y sequedad de boca con más frecuencia que quienes recibieron placebo ${ }^{5}$ (tabla 1 ), y que estos síntomas se vieron potenciados en el contexto del consumo de alcohol.

Por otro lado, la flibanserina puede causar hipotensión pronunciada, desmayos e interacciones con drogas como antimicóticos, antibióticos y otros antidepresivos, lo que no es menor dado que se debe tomar diariamente y por tiempo prolongado.

Tabla 1: comparación entre la frecuencia de efectos adversos entre

** Esto no implica que la industria farmacéutica se haya convertido en una enemiga de la humanidad, ya que ha tenido un rol importantísimo en el alivio de nuestro sufrimiento, en el control y/o la erradicación de muchas enfermedades y en la prolongación y mejora cualitativa de nuestra vida.

\begin{abstract}
"t Por ejemplo, si acudimos a un concesionario de automóviles porque queremos informarnos de las prestaciones y los precios de los diferentes modelos ofrecidos para tomar la mejor decisión posible de acuerdo a nuestro presupuesto, sabemos que el empleado de la concesionaria que nos va a atender obtendrá un beneficio económico si logra realizar la venta ("comisión"). Puede gustarnos o no que nos asesore alguien que se beneficiará si nosotros realizamos la compra, podemos decidir seguir o no sus consejos, pero por lo menos se trata de un contrato "blanqueado", que podemos aceptar o no. Vale destacar que no ocurre así en algunas relaciones con proveedores de salud, que también cobran una "comisión" por producto prescripto, por prótesis indicada, etc.; sin que este vínculo comercial sea transparente y declarado al cliente (en este caso el paciente).
\end{abstract}

${ }^{\ddagger}$ Carrera de Medicina del Instituto Universitario Hospital Italiano.

§ Servicio de Medicina Familiar y Comunitaria y Departamento de Investigación del Hospital Italiano de Buenos Aires. Departamento de Salud Pública del Instituto Universitario Hospital Italiano. sergio.terrasa@hiba.org.ar 
las mujeres que recibieron flibanserina y las asignadas a placebo.

\begin{tabular}{c|c|c} 
Efecto adverso & $\begin{array}{c}\text { Placebo } \\
(\mathbf{n}=\mathbf{1 . 5 5 6})\end{array}$ & $\begin{array}{c}\text { Flibanserina } \\
(\mathbf{n}=\mathbf{1 . 5 4 3})\end{array}$ \\
\hline Mareos & $2,2 \%$ & $11,4 \%$ \\
\hline Somnolencia & $2,9 \%$ & $11,2 \%$ \\
\hline Náuseas & $3,9 \%$ & $10,4 \%$ \\
\hline Fatiga & $5,5 \%$ & $9,2 \%$ \\
\hline Insomnio & $2,8 \%$ & $4,9 \%$ \\
\hline Boca seca & $1 \%$ & $2,4 \%$ \\
\hline
\end{tabular}

Fuente: FDA U.S food and drug administration. Drug Trials Snapshots: ADDYI (flibanserin). Disponible en URL:

http://www.fda.gov/Drugs/InformationOnDrugs/ucm462028.htm (último acceso: 29/11/2015).

Luego de que la aprobación de la droga fuera rechazada por segunda vez por la FDA, comenzó a hacer lobby una organización llamada "Igualar el marcador" (en inglés Even The Score, ver figura 1$)^{67}$, que reunía una gran cantidad de grupos feministas de Estados Unidos y contaba con apoyo financiero de la industria farmacéutica, reclamando que la droga fuese autorizada para la venta. Su argumento era que si los hombres disponían del sildenafil, era "discriminatorio" que las mujeres no contaran con una píldora para tratar la DSF. La presión de esta organización fue clave en la aprobación de la flibanserina por parte de la FDA.

Figura 1: logo de la organización Igualar el marcador ("Even The Score").
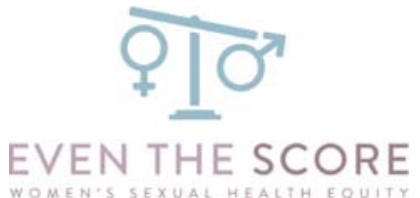

Una vez aprobada la droga, comenzó una campaña publicitaria dirigida directamente a las potenciales consumidoras, y al igual que el "Viagra" había entrado en la vida de los varones, estas comenzaron a demandar un fármaco que hacía un corto tiempo no sabían que "necesitaban", por un problema que no sabían que sufrían".

Vale aclarar que la fisiopatogenia de la DSF es compleja y debería entenderse en el marco de un enfoque biopsicosocial amplio. En su desarrollo existen componentes biológicos (por ejemplo el coito doloroso consecutivo a la atrofia vaginal de la menopausia) y psicosociales, como el estrés.
Las campañas de "concientización" y la pesquisa de personas medicalizables

Hacer visibles distintas problemáticas que se presentan en la vida cotidiana, convirtiéndolas en problemas de salud, es una herramienta ampliamente utilizada por la industria farmacéutica para aumentar sus ventas. Otra de las estrategias utilizadas incluye la difusión gratuita por Internet de cuestionarios autoadministrados con la supuesta finalidad de ayudar al médico a hacer el diagnóstico o a concientizar al paciente de su problema para solicitar ayuda. Actualmente está disponible en Internet el Cuestionario de Pesquisa del Deseo Sexual Hipoactivo, desarrollado por Boehringer Ingelheim Pharmaceuticals, Inc, que incluye preguntas como las siguientes: ¿En el pasado, su nivel de deseo sexual o interés era bueno y satisfactorio para usted? ¿Ha habido una disminución en su nivel de deseo o interés sexual? ¿Le gustaría que su nivel de deseo o interés sexual aumentara?

Podemos constatar que las preguntas incluidas en este cuestionario son muy poco específicas, lo que implica una alta probabilidad de que cualquier mujer sana responda al menos una pregunta en forma positiva, lo que puede promover su medicalización y aumentar la probabilidad de que le sea prescripto algún fármaco.

\section{Conclusión}

Podemos concluir que primero se detectó una situación social pasible de ser medicalizada (menor deseo sexual que el que se supone que es "normal"), para darle a continuación a este pro-blema un marco patológico o nosológico: disfunción sexual femenina.

Cuando se logró contar con una droga con una efectividad mínima y clínicamente cuestionable para tratar dicho trastorno (con efectos adversos no menospreciables), en una primera instancia este fármaco no fue aprobado por la FDA por estas razones. Sin embargo, la comunidad femenina comenzó a ser blanco de una campaña publicitaria para que reclamara el derecho a poder recibirlo, lo que condujo a que el fármaco fuera finalmente autorizado.

Si bien reconocemos que el avance de las tecnologías aplicadas a la salud promovido por la industria farmacéutica ha mejorado nuestra calidad y nuestra expectativa de vida, y que algunas mujeres con DSF pueden beneficiarse del uso de Flibanserina, consideramos que el caso que hemos descripto es un buen ejemplo de una estrategia de generación de nuevos nichos de mercado a través de la creación de una enorme cantidad de nuevas potenciales consumidoras

Agradecemos los comentarios y las sugerencias de los Dres: Karin Kopitowski y Eduardo Durante.

Recibido el 01/12/2015 y entregado el 20/12/2015.

\section{Referencias}

1. Association, A. P. Guía de consulta de los criterios diagnósticos del DSM-5. (Asociación Americana de Psiquiatría, 2014).

at <http://scholar.google.com/scholar?hl=en\&btnG=Search\&q=intitle:Gu a+de+consulta+de+los+Criterios+diagn sticos+del+DSM-5\#0>

2. Kingsberg, S. \& Woodard, T. Disfunción Sexual Femenina Con Enfoque en la Disminución del Deseo. Obstet Gynecol 477-86 (2015).

at <http://journals.Iww.com/greenjournal/Documents/Feb2015_Translation_Kingsberg.pdf>

3. Commissioner, O. of the. Press Announcements - FDA approves first treatment for sexual desire disorder.

at <http://www.fda.gov/NewsEvents/Newsroom/PressAnnouncements/ucm458734.htm>

4. Joint Meeting of the Bone Reproductive and Urologic Drugs (BRUDAC), A. C., And, the D. S. \& Committee, R. M. (DSaRM) A. Flibanserin. Proposed Indication: Treatment of hypoactive sexual desire disorder in premenopausal women. (2015).

at <http://www.fda.gov/downloads/AdvisoryCommittees/CommitteesMeetingMaterials/Drugs/DrugSafetyandRiskManagementAdvisoryCommittee/UCM449088.pdf>

5. Research Center for Drug Evaluation and. Drug Approvals and Databases - Drug Trials Snapshots: ADDYI. (2015). at <http://www.fda.gov/Drugs/lnformationOnDrugs/ucm462028.htm> 6. The Issue « Even The Score. at <http://eventhescore.org/the-problem/>

7. Cindy Pearson. Overdiagnosis and Women's Health: Challenges and Opportunites. in at <https://www.youtube.com/watch?v=GZijClGBno0>

8. Bär, N. Viagra femenino: la letra chica detrás de la pastilla rosa - 20.08.2015. at <http://www.lanacion.com.ar/1820647-viagra-femenino-la-letra-chica-detras-de-la-pastilla-rosa>

9. Boehringer Ingelheim Pharmaceuticals, Inc. ObGynalliance. Decreased Sexual Desire Screener Brief Diagnostic Assessment for Generalized, Acquired HSDD. 2010. at <http://www.obgynalliance.com/files/tsd/DSDS_Pocketcard.pdf> 